\title{
SPECT/CT arthrography of the wrist in ulnocarpal impaction syndrome
}

\author{
Tobias Krüger • Urs Hug • Martin W. Hüllner • \\ Florian Schleich • Patrick Veit-Haibach • \\ Urs von Wartburg • Klaus Strobel
}

Received: 27 October 2010 / Accepted: 16 December 2010/Published online: 12 January 2011

(C) Springer-Verlag 2011

Ulnar-sided wrist pain is a diagnostic challenge for hand surgeons and radiologists due to the complex anatomy of the involved small structures. Along with the clinical history and physical examination, imaging is important in the evaluation of ulnar-sided wrist pain [1-4]. Ulnocarpal impaction syndrome can lead to severe damage to the cartilage, triangular fibrocartilage complex (TFCC) and bone. The new generation of SPECT/CT devices allow SPECT and high-resolution CT imaging of the wrist with a spatial resolution of $0.33 \mathrm{~mm}$ in one imaging step [5]. CT arthrography of the wrist is a wellestablished investigation [6]. Combining wrist arthrography (arrowheads intraarticular contrast medium) and SPECT/CT (Arthro-SPECT/CT) offers a "one-stop shop" imaging approach for patients with ulnocarpal impaction.

In our 63-year-old male patient with ulnar-sided wrist pain for 3 months, Arthro-SPECT/CT demonstrated severe osteoarthritis of the distal radioulnar joint (DRUJ), profound damage to the cartilage of the ulnotriquetral joint (short arrow) and a large defect in the central part of the TFCC (short arrow). Concomitant increased uptake was observed in the triquetral bone (long arrow) and DRUJ. Arthro-SPECT/CT of the wrist is feasible and offers unique anatomic and metabolic information to the hand surgeon in patients with ulnar-sided wrist pain. Arthro-SPECT/CT seems to be a promising alternative to wrist MRI.

T. Krüger · M. W. Hüllner · F. Schleich · P. Veit-Haibach •

K. Strobel $(\bowtie)$

Department of Nuclear Medicine/Radiology,

Cantonal Hospital Lucerne,

Lucerne, Switzerland

e-mail: klaus.strobel@ksl.ch

$\mathrm{U}$. Hug $\cdot$ U. von Wartburg

Department of Hand and Plastic Surgery,

Cantonal Hospital Lucerne,

Lucerne, Switzerland

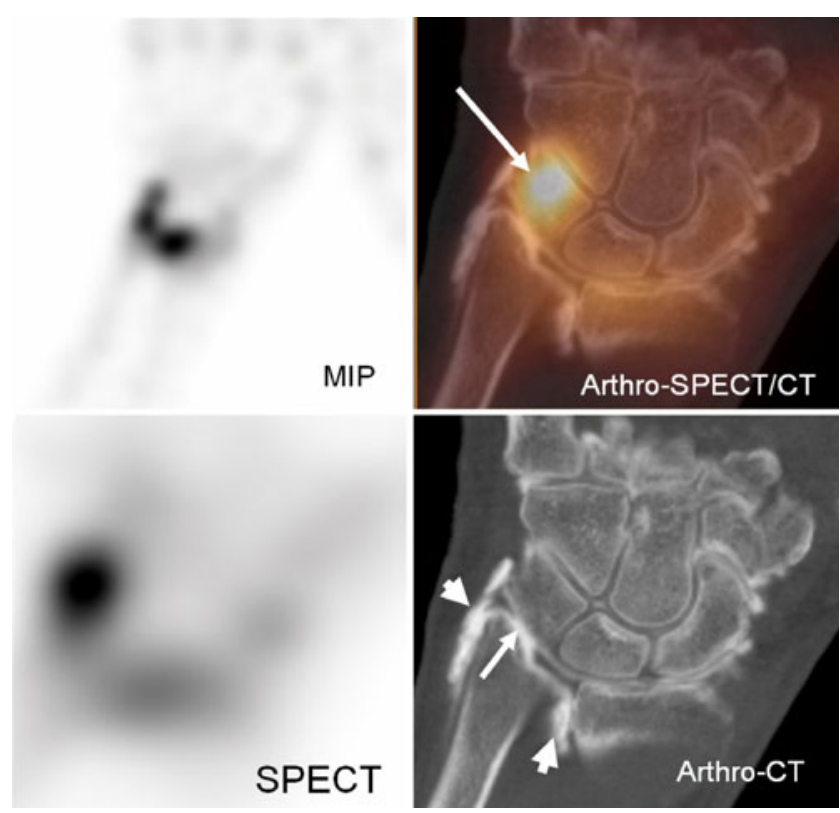

Conflicts of interest None.

\section{References}

1. Watanabe A, Souza F, Vezeridis PS, Blazar P, Yoshioka H. Ulnarsided wrist pain. II. Clinical imaging and treatment. Skeletal Radiol. 2010;39:837-57. doi: 10.1007/s00256-009-0842-3

2. Coggins CA. Imaging of ulnar-sided wrist pain. Clin Sports Med. 2006;25:505-26. doi: 10.1016/j.csm.2006.02.008.

3. Bickel KD. Arthroscopic treatment of ulnar impaction syndrome. J Hand Surg Am. 2008;33:1420-3. doi: 10.1016/j.jhsa.2008.07.014.

4. Cerezal L, del Pinal F, Abascal F, Garcia-Valtuille R, Pereda T, Canga A. Imaging findings in ulnar-sided wrist impaction syndromes. Radiographics. 2002;22:105-21.

5. Mariani G, Bruselli L, Kuwert T, Kim EE, Flotats A, Israel O, et al. A review on the clinical uses of SPECT/CT. Eur J Nucl Med Mol Imaging. 2010;37:1959-85. doi: 10.1007/s00259-010-1390-8.

6. Moser T, Dosch JC, Moussaoui A, Buy X, Gangi A, Dietemann JL. Multidetector CT arthrography of the wrist joint: how to do it. Radiographics. 2008;28:787-800. 